\title{
Type-2 Fuzzy VIKOR Method and Its Application in Facility Location Selection
}

\author{
Kuan Liu ${ }^{a}$, Yanwu Liu ${ }^{b}$ and Tao Jiang ${ }^{c}$ \\ School of Management, Wuhan University of Technology, Wuhan 430070, China \\ alk160513@163.com, blywwhut@163.com, 'cgofujiang@163.com
}

Keywords: Interval type-2 fuzzy decision making, VIKOR method, facility location selection.

\begin{abstract}
This study presents a multi-criteria group decision making (MCGDM) approach named interval type-2 fuzzy VIKOR method, which aims to help new enterprises determine facility locations in a more reliable way. Initially, decision makers (DMs) describe the alternative locations within the context of linguistic variables. Moreover, this paper models the values of these variables with interval type-2 fuzzy sets (IT2FSs). Finally, a compromise solution which is the 'closet' one to the ideal solution can be obtained through computing by VIKOR method under IT2FSs environment. A numerical example is given to demonstrate the validity of the presented method.
\end{abstract}

\section{Introduction}

Recently, decelerating economic growth in China has been bringing more challenges for enterprises in developing. For new manufacturing enterprises, they are faced with more drastic competitions. As the key step at the beginning, the determination of facility location is very important that it can directly influence late development of the enterprise. Therefore, to survive in the fierce competition, an enterprise must choose the most suitable facility location for further operation and development.

Facility location selection (FLS) is a multi-criteria group decision making (MCGDM) problem. Researchers have done some new work in relative areas in recent several years. Kahraman, Shuo-Yan and Shen, Chun-Ying et al. [1-3] proposed type-1 fuzzy methods to evaluate and select facility locations. Lin et al. [4] presented an approach on facility location selection based on the generalized interval 2-tuple linguistic Shapley chi-square averaging operator. Wang et al. [5] solved the FLS problem by using fuzzy-connective-based aggregation networks. Kannan et al. [6] aimed at prioritizing alternative potential locations for manufacturing firms with respect to economic, environmental, and societal dimensions.

However, as the FLS is very important, it is necessary to pay more attention to choosing the right location to keep the enterprise competitive. The process of solving FLS contains two stages: decision makers collect descriptions of potential locations from different dimensions the first stage; model and compute this information and then get the proposed location in the second stage. Compared with real numbers, the linguistic variables have more freedom in the aspect of semantic expression. IT2FSs can cover more uncertain factors than type-1 fuzzy sets (T1FSs) in modeling linguistic variables. The proposed location given by above methods are optimal solutions. But, sometimes, they might not be the most suitable location for the development of the enterprise. The potential locations have advantages in different aspect, they don't have the uniform object to compare with. Thus, motivated by these, this study aims to propose an interval type-2 fuzzy VIKOR method which concerns on finding the compromise solution that is the 'closet' one to the ideal solution. Hence, it can be sure that the final location determined is the most suitable location.

The rest of paper is organized as follows. Section 2 reviews some basic concepts and definitions of IT2FSs and the VIKOR decision method. Section 3 proposes the VIKOR method under interval type-2 fuzzy environment. Section 4 includes a simple numerical example to verify the proposed method. Finally, conducts conclusions from study in section 5. 


\section{Preliminaries}

\subsection{The concepts of T2FSs and IT2FSs}

Since originally proposed by Zadeh [7] in 1975, the type-2 fuzzy sets (T2FSs) were mainly developed by Mendel and al. The T2FSs are characterized by two membership functions that are primary membership function (PMF) and the second membership function (SMF).

Definition [8]. In the universe of discourse X, a T2FSs $\widetilde{\widetilde{A}}$ can be characterized by $\mu_{\widetilde{\mathrm{A}}}$, the type-2 membership function, expressed as follows:

$$
\widetilde{\widetilde{A}}=\left\{\left((x, u), \mu_{\widetilde{\mathrm{A}}}(\mathrm{x}, \mathrm{u})\right) \mid \forall \mathrm{x} \in \mathrm{X}, \forall \mathrm{u} \in[0,1]\right\},
$$

where $0 \leqq \mu_{\widetilde{\mathrm{A}}}(\mathrm{x}, \mathrm{u}) \leqq 1$ for any admissible $\mathrm{x}$ and $\mathrm{u}$.

In Eq.(1), when $\mu_{\widetilde{\mathrm{A}}}(\mathrm{x}, \mathrm{u})=1, \widetilde{\widetilde{\mathrm{A}}}$ is an IT2FSs which can be regarded as a special case of T2FSs. For simplifing the computation, we take trapezoidal interval type-2 fuzzy sets (TrIT2FSs) $\widetilde{\widetilde{A}}$ to represent IT2FSs.

$$
\widetilde{\widetilde{\mathrm{A}}}=\left(\widetilde{\mathrm{A}}^{+}, \widetilde{\mathrm{A}}^{-}\right)=\left(\left(\mathrm{a}_{1}^{+}, \mathrm{a}_{2}^{+}, \mathrm{a}_{3}^{+}, \mathrm{a}_{4}^{+} ; \mathrm{h}_{\widetilde{\mathrm{A}}}^{+}\right),\left(\mathrm{a}_{1}^{-}, \mathrm{a}_{2}^{-}, \mathrm{a}_{3}^{-}, \mathrm{a}_{4}^{-} ; \mathrm{h}_{\tilde{\mathrm{A}}}^{-}\right)\right) \text {, shown in Fig } 1 .
$$



Fig. $1 \mathrm{~A}$ trapezoidal interval type-2 fuzzy set

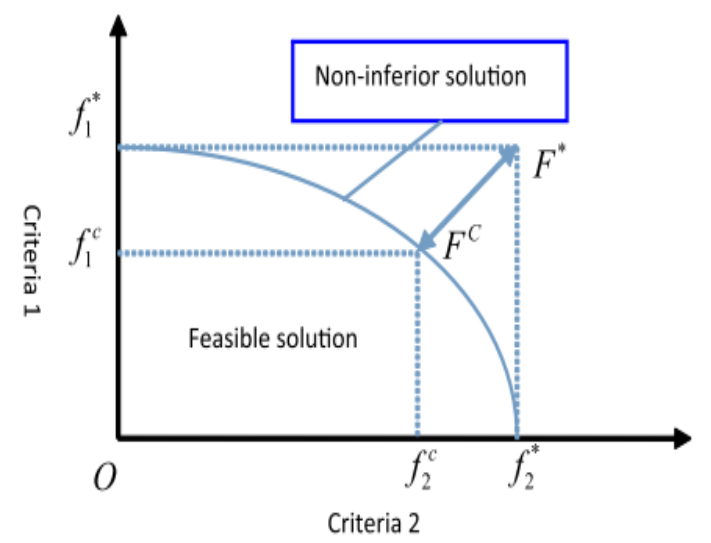

Fig. 2 The compromise solution of VIKOR.

Definition 2 [9]. Let $\widetilde{\widetilde{\mathrm{A}}}=\left(\widetilde{\mathrm{A}}^{+}, \widetilde{\mathrm{A}}^{-}\right)$and $\widetilde{\widetilde{\mathrm{B}}}=\left(\widetilde{\mathrm{B}}^{+}, \widetilde{\mathrm{B}}^{-}\right)$be two TrIT2FSs, the arithmetic operations are defined as follows:

(1) Addition operation

$$
\begin{aligned}
& \widetilde{\widetilde{A}} \oplus \widetilde{\widetilde{B}}=\left(\widetilde{\mathrm{A}}^{+}, \widetilde{\mathrm{A}}^{-}\right) \oplus\left(\widetilde{\mathrm{B}}^{+}, \widetilde{\mathrm{B}}^{-}\right) \\
= & \left(\left(\mathrm{a}_{1}^{+}+\mathrm{b}_{1}^{+}, \mathrm{a}_{2}^{+}+\mathrm{b}_{2}^{+}, \mathrm{a}_{3}^{+}+\mathrm{b}_{3}^{+}, \mathrm{a}_{4}^{+}+\mathrm{b}_{4}^{+} ; \min \left(\mathrm{h}_{\widetilde{\mathrm{A}}}^{+}, \mathrm{h}_{\widetilde{\mathrm{B}}}^{+}\right)\right),\right. \\
& \left(\mathrm{a}_{1}^{-}+\mathrm{b}_{1}^{-}, \mathrm{a}_{2}^{-}+\mathrm{b}_{2}^{-}, \mathrm{a}_{3}^{-}+\mathrm{b}_{3}^{-}, \mathrm{a}_{4}^{-}+\mathrm{b}_{4}^{-} ; \min \left(\mathrm{h}_{\widetilde{\widetilde{\mathrm{A}}}}, \mathrm{h}_{\widetilde{\widetilde{\mathrm{B}}}}^{-}\right)\right) .
\end{aligned}
$$

(2) Multiplication by real number operation

$$
\mathrm{k} \widetilde{\widetilde{A}}=\left(\begin{array}{c}
\left(\mathrm{ka}_{1}^{+}, \mathrm{ka}_{2}^{+}, \mathrm{ka}_{3}^{+}, \mathrm{ka}_{4}^{+} ; \min \left(\mathrm{h}_{\widetilde{\mathrm{A}}}^{+}, \mathrm{h}_{\widetilde{\mathrm{B}}}^{+}\right)\right), \\
\left(\mathrm{ka}_{1}^{-}, \mathrm{ka}_{2}^{-}, \mathrm{ka}_{3}^{-}, \mathrm{ka}_{4}^{-} ; \min \left(\mathrm{h}_{\widetilde{\widetilde{\mathrm{A}}}}, \mathrm{h}_{\widetilde{\widetilde{\mathrm{B}}}}^{\overline{\widetilde{\mathrm{A}}}}\right)\right)
\end{array}\right) \text {. }
$$

Definition 3 [10]. Distance between $\widetilde{\widetilde{A}}$ and $\widetilde{\mathrm{B}}$

$$
\begin{aligned}
& \mathrm{R}_{\mathrm{d}}(\widetilde{\widetilde{\mathrm{A}}}, \tilde{\tilde{1}})=\frac{1}{2 \mathrm{~h}_{\widetilde{\widetilde{\mathrm{A}}}}^{-} \mathrm{h}_{\widetilde{\widetilde{A}}}^{+}} \mathrm{h}_{\widetilde{\widetilde{\mathrm{A}}}}\left(\mathrm{a}_{4}^{+}-\mathrm{a}_{3}^{+}-\mathrm{a}_{4}^{-}+\mathrm{a}_{3}^{-}\right) \\
& -\frac{1}{2 \mathrm{~h}_{\widetilde{\mathrm{A}}}^{-} \mathrm{h}_{\widetilde{\mathrm{A}}}^{+}} \mathrm{h}_{\widetilde{\widetilde{\mathrm{A}}}}^{+}\left(0.5\left(\mathrm{a}_{2}^{-}-\mathrm{a}_{1}^{-}-\mathrm{a}_{2}^{+}+\mathrm{a}_{1}^{+}\right)-\left(\mathrm{a}_{4}^{-}-\mathrm{a}_{3}^{-}-\mathrm{a}_{2}^{-}+\mathrm{a}_{1}^{-}\right)\right) \\
& +1-\mathrm{a}_{4}^{-}-0.5\left(\mathrm{a}_{1}^{-}-\mathrm{a}_{1}^{+}+\mathrm{a}_{4}^{+}-\mathrm{a}_{4}^{-}\right) . \\
& \text {Where } \widetilde{\tilde{\tilde{1}}}=((1,1,1,1 ; 1),(1,1,1,1 ; 1)) . \\
& \mathrm{R}_{\mathrm{d}}(\widetilde{\widetilde{\mathrm{A}}}, \widetilde{\widetilde{\mathrm{B}}})=\left|\mathrm{R}_{\mathrm{d}}(\widetilde{\widetilde{\mathrm{A}}}, \tilde{\tilde{1}})-\mathrm{R}_{\mathrm{d}}(\widetilde{\widetilde{\mathrm{B}}}, \tilde{\tilde{1}})\right| .
\end{aligned}
$$

Definition 4. [11] Ranking value of $\widetilde{\widetilde{A}}$ 


$$
\begin{aligned}
& \operatorname{DTraIT}(\widetilde{\widetilde{A}})=\frac{1}{8}\left(\mathrm{a}_{4}^{+}-\mathrm{a}_{1}^{+}\right)+\left(\mathrm{h}_{\widetilde{\widetilde{\mathrm{A}}}}^{+} \cdot \mathrm{a}_{2}^{+}-\mathrm{a}_{1}^{+}\right)+\left(\mathrm{h}_{\widetilde{\widetilde{\mathrm{A}}}}^{+} \cdot \mathrm{a}_{3}^{+}-\mathrm{a}_{1}^{+}\right) \\
& +\frac{1}{8}\left(\mathrm{a}_{4}^{-}-\mathrm{a}_{1}^{-}\right)+\left(\mathrm{h}_{\widetilde{\widetilde{\mathrm{A}}}}^{\overline{\widetilde{A}}} \cdot \mathrm{a}_{2}^{-}-\mathrm{a}_{1}^{-}\right)+\left(\mathrm{h}_{\widetilde{\widetilde{\mathrm{A}}}}^{\bar{a}} \cdot \mathrm{a}_{3}^{-}-\mathrm{a}_{1}^{-}\right)+\frac{1}{2}\left(\mathrm{a}_{1}^{+}+\mathrm{a}_{1}^{-}\right) .
\end{aligned}
$$

\subsection{The VIKOR method}

The VIKOR method was proposed by Opricovic [12] and is considered as an efficient tool in finding the compromise solutions when solving MCGDM problems in which a set of conflicting criteria included. Fig. 2 shows that the compromise solution $F_{c}$ is a feasible solution that is the "closest" one to the ideal solution.

\section{The proposed fuzzy VIKOR method}

\subsection{The application of linguistic variables}

Compared with real numbers, words have more space to depict the emotional and semantic expression. This study chooses TrIT2FSs which contains ten parameters to model linguistic variables. Table 1 shows the interval typer-2 fuzzy scales of linguistic variables.

Table 1. Linguistic variables and interval typer-2 fuzzy scales

\begin{tabular}{cc}
\hline Linguistic variables & Trapezoidal interval type-2 fuzzy scales \\
\hline Very Poor $(\mathrm{VP})$ & $((0,0,0,0.1 ; 1),(0,0,0,0.5 ; 0.9))$ \\
Poor $(\mathrm{P})$ & $((0,0.1,0.2,0.3 ; 1),(0.05,0.1,0.15,0.2 ; 0.9))$ \\
Medium Poor $(\mathrm{MP})$ & $((0.1,0.3,0.4,0.5 ; 1),(0.2,0.3,0.35,0.4 ; 0.9))$ \\
Medium $(\mathrm{M})$ & $((0.3,0.5,0.6,0.7 ; 1),(0.4,0.5,0.55,0.6 ; 0.9))$ \\
Medium Good $(\mathrm{MG})$ & $((0.5,0.7,0.8,0.9 ; 1),(0.6,0.7,0.75,0.8 ; 0.9))$ \\
Good $(\mathrm{G})$ & $((0.7,0.9,0.95,1 ; 1),(0.8,0.85,0.9,0.95 ; 0.9))$ \\
Very Good $(\mathrm{VG})$ & $((0.9 .1 .1 .1 ; 1),(0.95,1,1,1 ; 0.9))$ \\
\hline
\end{tabular}

\subsection{The fuzzy VIKOR method}

Considering the decision makers $\mathrm{E}_{\mathrm{S}}(\mathrm{s}=1,2, \ldots, \mathrm{m})$ with respect to weight $\mathrm{w}_{\mathrm{S}}$ and the criterion $\mathrm{C}_{\mathrm{i}}(\mathrm{i}=1,2, \ldots, \mathrm{N})$ with respect to weight $\mathrm{w}_{\mathrm{i}}$, this study aims to select the most suitable facility location with the proposed method. The key steps of the fuzzy VIKOR method is shown as follows.

Step 1. Construct and aggregate the decision matrix

For each alternative location $A_{k}(k=1,2, \ldots, n)$, each decision maker $E_{s}$ give their evaluation with linguistic variables in all criteria dimensions. The fuzzy decision matrix $\widetilde{\widetilde{D}}_{\mathrm{E}_{\mathrm{s}}}$ constructed by decision makers can be expressed as Table 2:

\begin{tabular}{|c|c|c|c|c|c|c|c|c|c|c|}
\hline \multirow{2}{*}{ Criteria } & \multicolumn{3}{|c|}{$\widetilde{\widetilde{\mathrm{D}}}_{\mathrm{E}_{1}}$} & \multicolumn{3}{|c|}{$\widetilde{\widetilde{\mathrm{D}}}_{\mathrm{E}_{2}}$} & $\cdots$ & \multicolumn{3}{|c|}{$\widetilde{\widetilde{D}}_{\mathrm{E}_{\mathrm{m}}}$} \\
\hline & $\mathrm{A}_{1}$ & $\ldots$ & $A_{n}$ & $A_{1}$ & $\ldots$ & $A_{n}$ & $\ldots$ & $\mathrm{A}_{1}$ & $\ldots$ & $A_{n}$ \\
\hline $\mathrm{C}_{1}$ & $\tilde{\widetilde{\mathrm{d}}}_{1}^{11}$ & $\cdots$ & $\tilde{\tilde{\mathrm{d}}}_{1}^{\mathrm{n} 1}$ & $\tilde{\widetilde{\mathrm{d}}}_{1}^{12}$ & $\ldots$ & $\tilde{\tilde{d}}_{1}^{\mathrm{n} 2}$ & $\cdots$ & $\tilde{\tilde{\mathrm{d}}}_{\sim_{1}^{1 \mathrm{~m}}}^{1}$ & $\ldots$ & $\tilde{\tilde{\mathrm{d}}}_{\tilde{\sim}}^{\mathrm{nm}}$ \\
\hline $\mathrm{C}_{2}$ & $\tilde{\tilde{\mathrm{d}}}_{2}^{11}$ & $\ldots$ & $\tilde{\tilde{\mathrm{d}}}_{2}^{\mathrm{n} 1}$ & $\tilde{\tilde{\mathrm{d}}}_{2}^{12}$ & $\ldots$ & $\tilde{\tilde{\mathrm{d}}}_{2}^{\mathrm{n}} \mathrm{n}$ & $\ldots$ & $\tilde{\tilde{\mathrm{d}}}_{2}^{1 \mathrm{~m}}$ & $\ldots$ & $\tilde{\tilde{\mathrm{d}}}_{2}^{\mathrm{nm}}$ \\
\hline$\vdots$ & $\vdots$ & $\because$ & $\vdots$ & $\vdots$ & $\because$ & $\vdots$ & $\because$ & $\vdots$ & $\because$ & $\vdots$ \\
\hline $\mathrm{C}_{\mathrm{N}}$ & $\tilde{\tilde{\mathrm{d}}}_{\mathrm{N}}^{11}$ & $\ldots$ & $\tilde{\tilde{d}}_{\mathrm{N}}^{\mathrm{n} 1}$ & $\tilde{\tilde{\mathrm{d}}}_{\mathrm{N}}^{12}$ & $\ldots$ & $\tilde{\tilde{\mathrm{d}}}_{\mathrm{N}}^{\mathrm{n} 2}$ & $\ldots$ & $\tilde{\tilde{\mathrm{d}}}_{\mathrm{N}}^{1 \mathrm{~m}}$ & $\cdots$ & $\tilde{\tilde{\mathrm{d}}}_{\mathrm{N}}^{\mathrm{nm}}$ \\
\hline
\end{tabular}

Table 2. The fuzzy decision matrix

Each $\tilde{\mathrm{d}}_{\mathrm{i}}^{\mathrm{ks}}$ in the fuzzy decision matrix $\widetilde{\mathrm{D}}_{\mathrm{E}_{\mathrm{s}}}$ is an IT2FS. Before the next step, fuzzy decision matrix constructed by different DMs need to be aggregated. The weighted averages method is used in this process. In the aggregated fuzzy decision matrix, $\tilde{\tilde{\mathrm{d}}}_{\mathrm{i}}^{\mathrm{k}}=\sum_{\mathrm{s}=1}^{\mathrm{m}}\left(\mathrm{w}_{\mathrm{s}} * \tilde{\tilde{\mathrm{d}}}_{\mathrm{i}}^{\mathrm{ks}}\right)$.

Step 2. Select the best value and the worst value of each criterion

With respect to each criterion $C_{i}$, we choose one of the $\tilde{\tilde{d}}_{i}^{k}(k=1,2, \ldots, n)$ whose ranking value is the biggest as the best value $\tilde{\tilde{f}}_{i}^{*}$ and one of the $\tilde{\tilde{d}}_{i}^{k}(k=1,2, \ldots, n)$ whose ranking value is the smallest as the worst value $\tilde{\tilde{f}}_{\mathrm{i}}^{-}$. 
Step 3. Calculate the values of $S_{k}, R_{k}$ and $Q_{k}$ with the following relations:

$$
\begin{aligned}
& \mathrm{S}_{\mathrm{k}}=\sum_{\mathrm{i}=1}^{\mathrm{N}}\left(\mathrm{w}_{\mathrm{i}}^{*} \mathrm{~d}\left(\tilde{\tilde{\mathrm{f}}}_{\mathrm{i}}^{*}, \tilde{\tilde{\mathrm{d}}}_{\mathrm{i}}^{\mathrm{k}}\right) / \mathrm{d}\left(\tilde{\tilde{\mathrm{f}}}_{\mathrm{i}}^{*}, \tilde{\tilde{\mathrm{f}}}_{\mathrm{i}}^{-}\right)\right), \\
& \mathrm{R}_{\mathrm{k}}=\max _{\mathrm{i}}\left\{\mathrm{w}_{\mathrm{i}}^{*} \mathrm{~d}\left(\tilde{\tilde{\mathrm{f}}}_{\mathrm{i}}^{*}, \tilde{\tilde{\mathrm{d}}}_{\mathrm{i}}^{\mathrm{k}}\right) / \mathrm{d}\left(\tilde{\tilde{\mathrm{f}}}_{\mathrm{i}}^{*}, \tilde{\tilde{\mathrm{f}}}_{\mathrm{i}}^{-}\right)\right\}, \\
& \mathrm{Q}_{\mathrm{k}}=\lambda \frac{\mathrm{S}_{\mathrm{k}}-\mathrm{S}_{\mathrm{k}}^{-}}{\mathrm{S}_{\mathrm{k}}^{*}-\mathrm{S}_{\mathrm{k}}^{-}}+(1-\lambda) \frac{\mathrm{R}_{\mathrm{k}}-\mathrm{R}_{\mathrm{k}}^{-}}{\mathrm{R}_{\mathrm{k}}^{*}-\mathrm{R}_{\mathrm{k}}^{-}} .
\end{aligned}
$$

In Eq. (9), $\mathrm{S}_{\mathrm{k}}^{*}=\max _{\mathrm{k}}\left\{\mathrm{S}_{\mathrm{k}}\right\}, \mathrm{S}_{\mathrm{k}}^{-}=\min _{\mathrm{k}}\left\{\mathrm{S}_{\mathrm{k}}\right\}, \mathrm{R}_{\mathrm{k}}^{*}=\max _{\mathrm{k}}\left\{\mathrm{R}_{\mathrm{k}}\right\}, \mathrm{R}_{\mathrm{k}}^{-}=\min _{\mathrm{k}}\left\{\mathrm{R}_{\mathrm{k}}\right\}$ and the parameter $\lambda$ means the weight of the strategy of the maximum group utility. Considering the generality in using, assume that the value of $\lambda$ is set to 0.5 .

Step 4. Propose the alternative location

Rank the $Q_{k}, S_{k}$ and $R_{k}$ respectively from lower to higher. Before the alternative supplier $A^{1}$ with first position in the $\mathrm{Q}$ ranking proposed as the compromise solution, there are two conditions to satisfy.

Condition 1. Acceptable advantage:

$$
\mathrm{Q}\left(\mathrm{A}^{2}\right)-\mathrm{Q}\left(\mathrm{A}^{1}\right) \geq \frac{1}{\mathrm{n}-1}
$$

where $A^{2}$ means the alternative location with the second position in the $\mathrm{Q}$ ranking list, and $\mathrm{n}$ is the number of alternative suppliers.

Condition 2. Acceptable stability:

The alternative $A^{1}$ must also be the best in the $S$ and $R$ ranking list. If either condition is not satisfied, then go to the additional step to get the compromises solution:

When Condition 2 is not satisfied, then both $\mathrm{A}^{1}$ and $\mathrm{A}^{2}$ are compromise solutions.

When Condition 1 is not satisfied, the maximus value of $\mathrm{N}$ need to be explored with following relationship:

$$
Q\left(A^{N}\right)-Q\left(A^{1}\right)<\frac{1}{n-1} \text {. }
$$

Step 5. End.

\section{Numerical example}

In this section, we will identify the proposed method step by step.

The location must be located where maximum distribution and the minimum cost of production can be obtained. And then, other factors are also important such as room for expansion and safe living conditions for plant operation as well as the surrounding community. There are various criteria that must be considered while selecting a suitable location for an enterprise, but here, we have considered the following six attributes studied in [13], shown in Table 3.

Table 3 The criteria of FLS

\begin{tabular}{ccccccc}
\hline Criteria & $\mathrm{C}_{1}$ & $\mathrm{C}_{2}$ & $\mathrm{C}_{3}$ & $\mathrm{C}_{4}$ & $\mathrm{C}_{5}$ & $\mathrm{C}_{6}$ \\
& Skilled & Transport & Investment & Material & \multirow{2}{*}{ cost } \\
workers & facilities & resources & & $\begin{array}{c}\text { Expansion } \\
\text { possibility }\end{array}$ \\
\hline
\end{tabular}

Assume that the decision-making group contains three decision makers $E_{1}, E_{2}$ and $E_{3}$ with the value of weight $0.5,0.3$ and 0.2 , respectively. And the weight of $C_{i}(i=1,2, \ldots, 6)$ is $0.2,0.15,0.25$, $0.18,0.1$ and 0.12 , respectively. They need to choose one location as the facility location from five potential alternative locations $A_{k}(k=1,2, \ldots, 5)$.

Step 1. Three decision makers construct their own fuzzy decision matrix with rich experience in relative areas. Table 4 shows the decision matrix in detail. 
Table 4. The fuzzy decision matrix

\begin{tabular}{|c|c|c|c|c|c|c|c|c|c|c|c|c|c|c|c|}
\hline \multirow[b]{2}{*}{ C } & \multicolumn{5}{|c|}{$\widetilde{\widetilde{\mathrm{D}}}_{\mathrm{E}_{1}}$} & \multicolumn{5}{|c|}{$\widetilde{\widetilde{\mathrm{D}}}_{\mathrm{E}_{2}}$} & \multicolumn{5}{|c|}{$\widetilde{\widetilde{\mathrm{D}}}_{\mathrm{E}_{3}}$} \\
\hline & $\mathrm{A}_{1}$ & $\mathrm{~A}_{2}$ & $\mathrm{~A}_{3}$ & $\mathrm{~A}_{4}$ & $\mathrm{~A}_{5}$ & $A_{1}$ & $\mathrm{~A}_{2}$ & $\mathrm{~A}_{3}$ & $\mathrm{~A}_{4}$ & $\mathrm{~A}_{5}$ & $\mathrm{~A}_{1}$ & $\mathrm{~A}_{2}$ & $\mathrm{~A}_{3}$ & $\mathrm{~A}_{4}$ & $\mathrm{~A}_{5}$ \\
\hline $\mathrm{C}_{1}$ & $\mathrm{M}$ & MG & $\mathrm{G}$ & $\mathrm{P}$ & $\mathrm{M}$ & $\mathrm{M}$ & $\mathrm{M}$ & $\mathrm{MG}$ & MP & MG & $M$ & $\mathrm{G}$ & VG & M & $\mathrm{M}$ \\
\hline $\mathrm{C}_{2}$ & MP & $\mathrm{G}$ & $\mathrm{MG}$ & $\mathrm{M}$ & $\mathrm{MG}$ & MP & $\mathrm{P}$ & $\mathrm{G}$ & $\mathrm{M}$ & $\mathrm{G}$ & $\mathrm{M}$ & $\mathrm{G}$ & $\mathrm{MG}$ & MP & MG \\
\hline $\mathrm{C}_{3}$ & $\mathrm{M}$ & $\mathrm{P}$ & $\mathrm{G}$ & MP & $\mathrm{M}$ & $\mathrm{G}$ & $\mathrm{M}$ & MP & $\mathrm{G}$ & MP & MG & $\mathrm{M}$ & MP & G & MP \\
\hline $\mathrm{C}_{4}$ & $\mathrm{MG}$ & M & $\mathrm{M}$ & $\mathrm{G}$ & MP & MP & $\mathrm{G}$ & MG & $M$ & $\mathrm{M}$ & $\mathrm{MG}$ & $\mathrm{P}$ & $\mathrm{G}$ & $\mathrm{P}$ & $\mathrm{M}$ \\
\hline $\mathrm{C}_{5}$ & $\mathrm{G}$ & MP & $\mathrm{P}$ & $\mathrm{MG}$ & $\mathrm{M}$ & VP & $\mathrm{G}$ & $M$ & $\mathrm{P}$ & MP & VP & $\mathrm{M}$ & $\mathrm{P}$ & $\mathrm{M}$ & MP \\
\hline $\mathrm{C}_{6}$ & $\mathrm{P}$ & $\mathrm{G}$ & $\mathrm{M}$ & $\mathrm{M}$ & MP & $\mathrm{G}$ & MP & MG & MP & $\mathrm{MG}$ & MP & $\mathrm{MG}$ & $\mathrm{M}$ & MG & $\mathrm{P}$ \\
\hline
\end{tabular}

Step 2. Defuzzy the aggregated decision matrix by Eq. (6), we can obtain the best value and the worst value though comparison among them, shown in Table 5.

Table 5. The best value and the worst value

\begin{tabular}{|c|c|c|}
\hline Criteria & The best value $\tilde{\tilde{f}}_{\mathrm{i}}^{*}$ & The worst value $\tilde{\tilde{f}}_{\mathrm{i}}^{-}$ \\
\hline $\mathrm{C}_{1}$ & $\begin{array}{c}((0.6,0.8,0.88,0.95 ; 1),(0.7,0.78,0.82,0.88 \\
0.9)\end{array}$ & $\begin{array}{c}((0.05,0.2,0.3,0.4 ; 1),(0.12,0.2,0.25, \\
0.3 ; 0.9)\end{array}$ \\
\hline $\mathrm{C}_{2}$ & $\begin{array}{c}((0.6,0.8,0.88,0.95 ; 1),(0.7,0.78,0.82,0.88 \\
0.9))\end{array}$ & $\begin{array}{c}((0.1,0.3,0.4,0.5 ; 1),(0.2,0.3,0.35 \\
0.4 ; 0.9))\end{array}$ \\
\hline $\mathrm{C}_{3}$ & $\begin{array}{c}((0.5,0.7,0.78,0.85 ; 1),(0.6,0.68,0.72,0.78 \\
0.9))\end{array}$ & $\begin{array}{c}((0.15,0.3,0.4,0.5 ; 1),(0.22,0.3,0.35 \\
0.4 ; 0.9))\end{array}$ \\
\hline $\mathrm{C}_{4}$ & $((0.5,0.7,0.8,0.9 ; 1),(0.6,0.7,0.75,0.8 ; 0.9))$ & $\begin{array}{c}((0.4,0.6,0.7,0.8 ; 1),(0.5,0.6,0.65 \\
0.7 ; 0.9))\end{array}$ \\
\hline $\mathrm{C}_{5}$ & $\begin{array}{c}((0.4,0.6,0.68,0.75 ; 1),(0.5,0.58,0.62,0.68 \\
0.9))\end{array}$ & $\begin{array}{c}((0.15,0.3,0.4,0.5 ; 1),(0.22,0.3,0.35, \\
0.4 ; 0.9))\end{array}$ \\
\hline $\mathrm{C}_{6}$ & $((0.4,0.6,0.7,0.8 ; 1),(0.5,0.6,0.65,0.7 ; 0.9))$ & $((0.2,0.4,0.5,0.6 ; 1),(0.3,0.4,0.45,0.5 ; 0.9))$ \\
\hline
\end{tabular}
6:

Step 3. Calculate the values of $S_{k}, R_{k}$ and $Q_{k}$ by using Eqs. (7-9), values are presented in Table

Table 6 Values of $S_{k}, R_{k}$ and $Q_{k}$

\begin{tabular}{cccc}
\hline Alternative locations & $\mathrm{S}_{\mathrm{k}}$ & $\mathrm{R}_{\mathrm{k}}$ & $\mathrm{Q}_{\mathrm{k}}$ \\
\hline $\mathrm{A}_{1}$ & 0.78 & 0.36 & 0.68 \\
$\mathrm{~A}_{2}$ & 0.5 & 0.25 & 0.25 \\
$\mathrm{~A}_{3}$ & 0.35 & 0.18 & 0 \\
$\mathrm{~A}_{4}$ & 0.6 & 0.2 & 0.28 \\
$\mathrm{~A}_{5}$ & 0.85 & 0.54 & 1 \\
\hline
\end{tabular}

Step 4. Propose the compromise solution

Rank the alternative locations, sorting by the values $S_{k}, R_{k}$ and $Q_{k}$ in ascending order. The results are three ranking lists as follows: $S_{3}<S_{2}<S_{4}<S_{1}<S_{5} ; R_{3}<R_{4}<R_{2}<R_{1}<R_{5} ; Q_{3}<$ $\mathrm{Q}_{2}<\mathrm{Q}_{4}<\mathrm{Q}_{1}<\mathrm{Q}_{5} \cdot \mathrm{Q}\left(\mathrm{A}^{2}\right)-\mathrm{Q}\left(\mathrm{A}^{1}\right)=0.25 \geq \frac{1}{5-1}$. It is apparently that proposing $\mathrm{A}_{3}$ as the compromise solution satisfies two conditions.

Step 5. End.

\section{Conclusions}

This study introduced an interval type-2 fuzzy VIKOR method which aims to find the most suitable facility location for enterprises. The linguistic variables can depict the semantic expressions in a better way. Furthermore, model the linguistic variables with IT2FSs can capture more uncertain factors in decision making. The compromise solution given by this method is the 'closet' one to the ideal solution. And, the numerical example identified the validity of the proposed method. The result shows this method is efficient and can be used in solving MCGDM problems. 


\section{Acknowledgments}

This work was financially supported by the Independent Innovation Foundation of Wuhan University of Technology under the Project No. 2016VI034 and the Fundamental Research Funds for the Central Universities under the Project No. 2015VI002.

\section{References}

[1]. Kahraman, C., D. Ruan, and I. Dogan, Fuzzy group decision-making for facility location selection. Information Sciences, 2003. 157: pp. 135-153.

[2]. Chou, S.-Y., Y.-H. Chang, and C.-Y. Shen, A fuzzy simple additive weighting system under group decision-making for facility location selection with objective/subjective attributes. European Journal of Operational Research, 2008. 189(1): pp. 132-145.

[3]. Shen, C.-Y. And K.-T. Yu, A generalized fuzzy approach for strategic problems: The empirical study on facility location selection of authors' management consultation client as an example. Expert Systems with Applications, 2009. 36(3, Part 1): pp. 4709-4716.

[4]. Lin, J., Q. Zhang, and F. Meng, An approach for facility location selection based on optimal aggregation operator. Knowledge-Based Systems, 2015. 85: pp. 143-158.

[5]. Wang, F.-F., L.-F. Chen, and C.-T. Su, Location selection using fuzzy-connective-based aggregation networks: a case study of the food and beverage chain industry in Taiwan. Neural Computing and Applications, 2015. 26(1): pp. 161-170.

[6]. Govindan, K., et al., Effect of product recovery and sustainability enhancing indicators on the location selection of manufacturing facility. Ecological Indicators, 2016. 67: pp. 517-532.

[7]. Zadeh, L.A., The concept of a linguistic variable and its application to approximate reasoningI. Information sciences, 1975. 8(3): pp. 199-249.

[8]. Mendel, J.M., M.R. Rajati, and P. Sussner, on clarifying some definitions and notations used for type-2 fuzzy sets as well as some recommended changes. Information Sciences, 2016. 340-341: pp. 337-345.

[9]. Chen, S.-M., et al., Fuzzy multiple attributes group decision-making based on ranking interval type-2 fuzzy sets. Expert Systems with Applications, 2012. 39(5): pp. 5295-5308.

[10]. Qin, J., X. Liu, and W. Pedrycz, An extended VIKOR method based on prospect theory for multiple attribute decision making under interval type-2 fuzzy environment. Knowledge-Based Systems, 2015. 86: pp. 116-130.

[11]. Kahraman, C., et al., Fuzzy analytic hierarchy process with interval type-2 fuzzy sets. Knowledge-Based Systems, 2014. 59: pp. 48-57.

[12]. Opricovic, S., Multicriteria optimization of civil engineering systems. Faculty of Civil Engineering, Belgrade, 1998. 2(1): pp. 5-21.

[13]. Devi, K. and S.P. Yadav, A multicriteria intuitionistic fuzzy group decision making for plant location selection with ELECTRE method. The International Journal of Advanced Manufacturing Technology, 2013: pp. 1-11. 